\title{
Leuconostoc mesenteroides
}

National Cancer Institute

\section{Source}

National Cancer Institute. Leuconostoc mesenteroides. NCI Thesaurus. Code C86497.

A species of facultatively anaerobic, gram positive, cocci shaped bacteria in the phylum Firmicutes. This species is nonmotile, catalase and oxidase negative, produces acid from arabinose, raffinose and trehalose and hydrolyzes esculin. L. mesenteroides is usually nonpathogenic and is used as a starter culture for the fermentation of cabbage during sauerkraut production. 